\title{
A.C. Loss in a Stack of Flat Superconducting Cables
}

\author{
Alexander A. Akhmetov
}

\begin{abstract}
An equation has been derived which describes the current distribution in a flat cable subjected to a time-dependent magnetic field directed perpendicular to the cable wide face. Solutions of this equation obtained in the case when all parameters are uniform along the cable allow us to obtain the time constant spectrum, magnetic moment of the cable and A.C. loss. For a stack of cables with height much larger than the cable width, the contribution of the self-screening is calculated analytically. Numerical examples are provided based on geometrical characteristics and interstrand contact resistance of a typical LHC cable and the inductance of the LHC dipole.
\end{abstract} bles.

Index Terms-A.C. loss, coupling currents, superconducting ca-

\section{INTRODUCTION}

$\mathbf{F}$ OR THE last decade lumped circuit models [1]-[6] have been developed to describe eddy currents in a flat superconducting $K$-strand cable subjected to background magnetic field variations. These models divide each strand of the cable into $M \gg 1$ finite elements. The elements belonging to different strands are connected via lumped resistances. The magnetic field density in the vicinity of these elements is discretized and represented in the calculations by the finite array of numbers of rank $Q$, where $Q \gg K, Q \gg M$. Due to its size, the resulting set of $Q$ Faraday equations often has to be written in matrix form [2], [3].

As an alternative, the cable strands are considered as continuous entities. $K$ differential one-dimensional equations describe currents in the strands as functions of time and position along the cable [7], [8] while inter-strand resistances are smeared along the cable. These equations are also treated in matrix form, sometimes in combination with FEM [8]. The present paper attempts to show that the transition to a totally continuous model is possible. It results in a single 2-D equation describing the current distribution in a flat cable subjected to a time-dependent magnetic field directed perpendicular to the cable wide face.

\section{BASIC EQUATION}

Fig. 1 shows a schematic rendering of a flat cable. Solid lines ascending from the left to the right represent the strands of one (say, the front) layer. The other strands form the rear layer. The filled circles represent physically present electrical contacts with the resistance $R$ between layers. The other types of circles show fictional contacts. The nonfilled circles placed at the cable

Manuscript received September 24, 2001.

The author is with CERN, European Organization for Nuclear Research, CH-1211 Geneva 23, Switzerland on leave from the Institute for High Temperatures, Ac. of Science of Russia, Izhorskaya 13/19, Moscow, 127412 Russia (e-mail: alexandre.akhmetov@cern.ch).

Publisher Item Identifier S 1051-8223(02)04245-8.

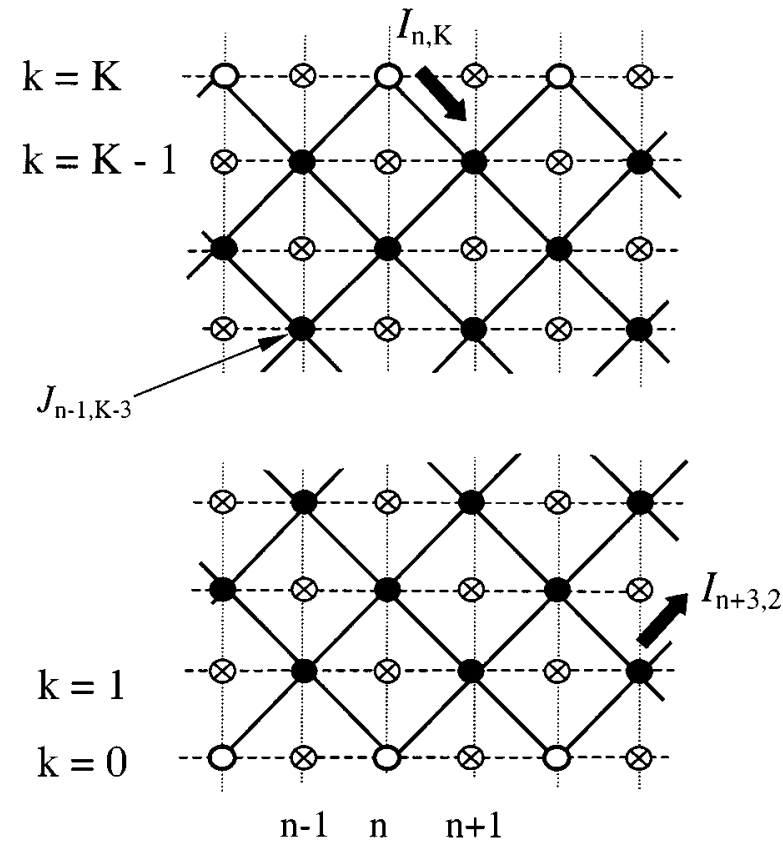

Fig. 1. A schematic view of a flat two-layer cable. Contacts marked $\bigcirc$ and $\otimes$ are fictional.

edges symbolize the contacts which are absent in the real cables. Instead, strands go around the edge from one layer to the other without any resistance. However, for the following it is convenient to assume that there are contacts, which carry no current [9].

Other fictional contacts marked $\otimes$ have no connection to the strands. They are placed in the center of elementary cells created by two adjacent strands of the front layer placed over two adjacent strands of the rear layer. Indexes $1 \leq k \leq K-1$ and $1 \leq n \leq N, N \gg 1$ are used to identify the cell position across and along the cable correspondingly. The same indexes are used to refer to the contact resistances and the strand portions located between two adjacent contacts. The cell marked $(n, k)$ is bounded by strand fragments $(n-1, k),(n-1, k+1),(n, k)$ and $(n, k+1)$ and contact resistances $(n-1, k),(n, k-1)$, $(n, k+1)$ and $(n+1, k)$ of the type $\bullet$ or $\circ$. The fictional contact $\otimes$ is placed in the center of the cell with the same indexes.

Let us apply the Faraday equation to the cell. We obtain

$$
\begin{aligned}
& R\left(J_{n-1, k}-2 J_{n, k}+J_{n+1, k}\right)-R\left(J_{n, k+1}-2 J_{n, k}+J_{n, k-1}\right) \\
& +L \frac{d}{d t}\left(I_{n-1, k+1}-I_{n-1, k}+I_{n, k+1}-I_{n, k}\right) \\
& \quad=\frac{l_{p} w}{K(K-1)} \frac{d B_{n, k}}{d t}
\end{aligned}
$$

where $R$ is the contact resistance, $J$ is the current across the contact resistance, $L$ is the inductance of the strand portion, $t$ 
is the time, $I$ is the current in the strand portion, $l_{p}$ is the cable twist pitch length, $w$ is the cable width and $B$ is the average magnetic field in the cell. The current $J_{n, k}$ across the fictional contact $\otimes$ is both added and deducted twice and is, therefore, cancelled.

Now, assuming $K \gg 1$, the scale of the spatial variations across the cable is $\Delta y=w / K \ll w$. Since the strand goes from one edge of the cable to the other one over the length $l_{p} / 2$, the scale of the spatial variations along the cable is $\Delta x=$ $l_{p} /(2 K) \ll l_{p}$. In the limit $\Delta x \rightarrow 0, \Delta y \rightarrow 0$, (1) becomes

$$
\frac{l_{p}^{2}}{4 w^{2}} \frac{\partial^{2} J}{\partial x^{2}}-\frac{\partial^{2} J}{\partial y^{2}}+\frac{L K^{2}}{R w^{2}} \frac{\partial}{\partial t}\left(\delta I_{f}+\delta I_{r}\right)=\frac{l_{p}}{R w} \frac{\partial B}{\partial t},
$$

where the magnetic field $B$ is due to both an external source $\left(B_{e}\right)$ and the strand currents $\left(B_{s}\right), B=B_{e}+B_{s}$, the $x$ axis and the $y$ axis are directed along and across the cable correspondingly, $\delta I_{f}$ and $\delta I_{r}$ are the variations of the currents in the front and rear layers with the ordinate increase by $\Delta y$. Equation (2) should be complimented by the boundary conditions $J(x, \pm s)=0, I_{f}( \pm l, y)=I_{r}( \pm l, y)=0$, where the origin of the coordinate is placed in the cable center, $s=w / 2$ and $l$ is the half of the sample length.

\section{EDDY CURRENT OSCILLATIONS}

Oscillations of eddy currents under stationary conditions are already well described [3], [5], [6]. Let us show that (2) provides a solution in a much simpler way. In stationary conditions it becomes

$$
\frac{l_{p}^{2}}{4 w^{2}} \frac{\partial^{2} J}{\partial x^{2}}-\frac{\partial^{2} J}{\partial y^{2}}=\frac{l_{p}}{R w} \frac{d B_{e}}{d t} .
$$

Let us assume that $d B_{e} / d t=$ constant, uniform across and along the cable. Solution of (3) satisfying symmetric boundary conditions $J(x, \pm s)=0, J(-l, y)=J(+l, y)$ is

$$
J=\frac{l_{p}}{2 R w} \frac{d B_{e}}{d t}\left(s^{2}-y^{2}\right)+D \cos \left(\frac{2 \pi}{l_{p}} x\right) \cos \left(\frac{\pi}{w} y\right),
$$

where $D$ is the constant. To find it, let us use the fact that in linear systems the correct current distribution should provide a minimum energy dissipation [3], [6]. As a result, we have

$$
D=-\frac{16 w l_{p}^{2}}{R \pi^{3}} \sin \left(2 \pi l / l_{p}\right)\left[4 \pi l+l_{p} \sin \left(4 \pi l / l_{p}\right)\right]^{-1} \frac{d B_{e}}{d t} .
$$

The coefficient $D$ of the eddy current oscillations is zero when $2 l=m l_{p}$, where $m$ is an integer, and reaches local maximums at $2 l=(m+1 / 2) l_{p}$. With $m$ increasing these maximums decrease.

\section{UNIFORM CURRENT DISTRIBUTION}

If $l \gg l_{p}$, the inter-strand currents $J$ become uniform along the cable, $I_{f}=I_{r}=I$ so that except for the case of extremely slow ramp rate, $J=(d I / d y) \Delta y$, and (2) takes the form [9]

$$
\frac{\partial^{2} J}{\partial y^{2}}-\frac{2 L K^{2}}{R w^{2}} \frac{\partial J}{d t}=-\frac{l_{p}}{R w} \frac{\partial B}{\partial t} .
$$

Let us consider not a single cable but a high stack of electrically insulated cables. In this case, a magnetic field created by screening currents in the position $y$ is

$$
B_{s}=\frac{2 \mu_{0} K}{w h} \int_{-s}^{y} I(y) d y,
$$

where $\mu_{0}$ is the magnetic permeability of vacuum and $h$ is the distance between the cables in the vertical direction $z$.

Combining (6) and (7), we have

$$
\frac{\partial^{3} I}{\partial y^{3}}-\frac{2 L K^{2}}{R w^{2}} \frac{\partial^{2} I}{\partial y \partial t}=-\frac{l_{p} K}{R w^{2}} \frac{\partial}{\partial t}\left(B_{0}+\frac{2 \mu_{0} K}{w h} \int_{-s}^{y} I d y\right) .
$$

Last, in the harmonic case when $B_{e}=B_{0} \exp (i \omega t)$ it is possible to separate the variables. Let $I=\varphi \cdot \exp (i \omega t)$. Then

$$
\frac{d^{3} \varphi}{d y^{3}}-i \omega \delta \frac{d \varphi}{d y}+i \omega \gamma \int_{-s}^{y} \varphi d y=-i \omega d
$$

where

$$
\delta=\frac{2 L K^{2}}{R w^{2}}, \quad \gamma=\frac{2 \mu_{0} K^{2} l_{p}}{R w^{3} h}, \quad d=\frac{K l_{p} B_{0}}{R w^{2}} .
$$

The relation between parameters $\delta$ and $\gamma$ underline the physics of the process. If $\delta \ll \gamma w^{2}$ then the influence of screening currents is prevailing over the inductance influence and vice versa.

Once the current distribution is found, the magnetic moment of a cable per twist pitch is

$$
M_{p}=-\frac{2 K l_{p}}{w} \exp (i \omega t) \int_{0}^{s} y \varphi d y,
$$

and the loss per cycle per twist pitch is

$$
G=\frac{2 \pi R K^{2}}{\omega w} \int_{0}^{s} J_{0}^{2} d y
$$

where $J_{0}=(w / K)(d \varphi / d y)$ is the amplitude of the oscillating current $J$.

\section{Loss Versus FREQUENCY DEPENDENCE}

Instead of solving (9) for arbitrary $\omega$, let us consider two limits; $\omega \rightarrow 0$ and $\omega \rightarrow \infty$. In the first case an influence of both strand inductances and screening currents can be neglected and the strand currents are given by [4], [9]

$$
I=\frac{l_{p} K}{6 R w^{2}} \frac{d B_{e}}{d t}\left(3 s^{2} y-y^{3}\right),
$$

providing a magnetic moment

$$
M_{p}=-\frac{w^{2} K^{2} l_{p}^{2}}{120 R} \frac{d B_{e}}{d t} .
$$

At the high frequency limit except for the narrow region at the cable edges [9], resistive terms in (9) can be neglected and we have

$$
\frac{d^{2} \varphi}{d x^{2}}-\alpha^{2} \varphi=0
$$


where $\alpha^{2}=\gamma \delta^{-1}$. Its solution satisfying the boundary conditions is

$$
\varphi=\frac{l_{p} B_{0}}{2 L K \alpha} \frac{\operatorname{sh}(\alpha y)}{\operatorname{ch}(\alpha s)}
$$

from which we get a magnetic moment at $\omega \rightarrow \infty$ as

$$
M_{p} \rightarrow-\frac{l_{p}^{2} B_{0}}{w L \alpha^{2} \operatorname{ch}(\alpha s)}\left(s \cdot \operatorname{ch}(\alpha s)-\frac{\operatorname{sh}(\alpha s)}{\alpha}\right) \exp (i \omega t) .
$$

The values given by (14) and (17) can be fitted by the general expression

$$
M_{p}=-\frac{i n_{s} \tau_{s} \omega B_{0}}{\mu_{0}\left(1+i \omega \tau_{s}\right)} \exp (i \omega t)
$$

where

$$
n_{s}=l_{p} h \frac{s \alpha-\operatorname{th}(\alpha s)}{\alpha}, \quad \tau_{s}=\frac{\mu_{0} K^{2} l_{p} w^{2}}{120 R h} \frac{\alpha}{s \alpha-\operatorname{th}(\alpha s)} .
$$

Now, following arguments of [10], let us write loss versus frequency dependence in the form

$$
G=\frac{n_{s} \tau_{s} \pi \omega B_{0}^{2}}{\mu_{0}\left(1+\omega^{2} \tau_{s}^{2}\right)}
$$

Equation (20) indicates that at low frequencies the loss increases linearly with frequency, reaches the maximum at $\omega=1 / \tau_{s}$ and decreases afterwards.

\section{TIME CONSTANT SPECTRUM}

It is known that in nonuniform conditions the time constants of the flat cable can be very large [6]-[8], [11]. This is not the case when both the cable and applied magnetic field are uniform along the cable length. To find the time constants, let us analyze the decay of currents induced in the cable up to the moment $t=t_{0}$ if $d B_{e} / d t=0$ at $t \geq t_{0}$. The correspondingly reduced (2) has the form

$$
\frac{\partial^{3} I}{\partial y^{3}}-\delta \frac{\partial^{2} I}{\partial y \partial t}+\gamma \frac{\partial}{\partial t} \int_{-s}^{y} I d y=0
$$

Let us separate variables by using

$$
I(y, t)=\chi(y) \exp \left(-t / \tau_{m}\right),
$$

which leads to the following equation

$$
\tau_{m} \frac{d^{3} \chi}{d y^{3}}+\delta \frac{d \chi}{d y}-\gamma \int_{-s}^{y} \chi d y=0
$$

with the evident solution

$$
\chi=A_{\nu} \cdot \sin (\nu y),
$$

where $A_{\nu}$ is the coefficient depending on the current distribution at $t=t_{0}$ and $\nu$ is the coefficient, which must satisfy boundary conditions. Substituting this solution in (23) and using the above boundary conditions we have

$$
\tau_{m} \nu^{4}-\delta \nu^{2}-\gamma=0
$$

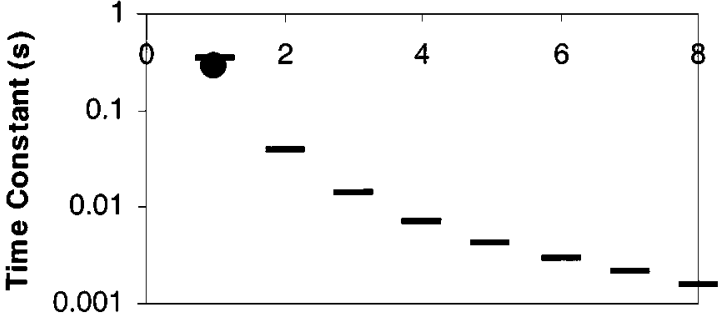

Time Constant Number

Fig. 2. Time constants $\tau_{m}$ (-) versus number $m$ for the screening prevailing The $\tau_{1} \approx \tau_{s}(\bullet)$, the other $\tau_{m}$ are much smaller. Correspondingly, the coupling currents given by (24) decay fast and can be important at high frequencies only.

and, via $\nu s=\pi / 2,3 \pi / 2,5 \pi / 2 \ldots$, or $\nu_{m}=\pi(2 m-1) / w$, we define the discrete values allowed for $\tau_{m}$ as

$$
\tau_{m}=\left[w^{2} \delta \pi^{2}(2 m-1)^{2}+\gamma w^{4}\right] \pi^{-2}(2 m-1)^{-4},
$$

where $m=1,2 \ldots$. Formally, in the continuous model there is no upper limit for $m$. However, (2) is correct only if the spatial variations governed by (24) occur on a length much longer than $\Delta y$. Hence, only a limited number of time constants has to be taken into account.

Let us consider the first time constant of the spectrum $\tau_{1}$ in the limit of screening prevailing. Below, $\tau_{1}$ is shown in comparison with the time constant $\tau_{s}$ defining the maximum of the loss curve. The last one is given in the same limit when $\operatorname{th}(\alpha s) \ll \alpha s$ :

$$
\tau_{1}=\frac{2 \mu_{0} l_{p} w K^{2}}{\pi^{4} R h}, \quad \tau_{s}=\frac{\mu_{0} l_{p} w K^{2}}{60 R h} .
$$

It is seen that these values are quite close.

\section{LHC InNER CABLE TIME CONSTANT Estimation}

Let us estimate the time constant for the high stack composed of the LHC dipole inner cables with $K=28, w=15 \mathrm{~mm}, l_{p}=$ $11.5 \mathrm{~mm}$ and the mid-thickness equal to $1.9 \mathrm{~mm}$. Allowing for the inter-cable insulation let us take $h=2 \mathrm{~mm}$. For the $L$ value let us take first the lowest estimation based on the magnetic energy associated with one strand [11]. If $l_{f}=18 \mathrm{~mm}$ is the strand twist pitch, $d=1.07 \mathrm{~mm}$ is the strand diameter and $d_{c}=$ $0.65 \mathrm{~mm}$ is the average diameter of the multi-filamentary area in the Alstom-made strand [12] then

$$
L=\frac{\mu_{0} \sqrt{l_{p}^{2}+4 w^{2}}}{4 K}\left[\frac{\pi d_{c}^{2}}{2 l_{f}^{2}}+\frac{1}{\pi} \ln \left(\frac{d}{d_{c}}\right)\right] .
$$

Putting the above values in (28) we have $L \approx 2.1 \cdot 10^{-10} \mathrm{H}$. The average contact resistance in the inner windings of pre-series dipoles is $R \approx 50 \mu \Omega$ [13], which give us parameter $\alpha \approx$ $4800 \mathrm{~m}^{-1}$. Now, it is possible to estimate a time constant, which is $\tau_{s} \approx 0.3 \mathrm{~s}$ (see Fig. 2). The value $\alpha w \approx 70 \gg 1$ corresponds to a case where the contribution from the screening to the time constants is much higher than the inductance contribution. Calculations using (28) can be thought of as an underestimation of $L$. However, even for $L$ being two orders of magnitude higher, i.e., for $L=2 \cdot 10^{-8} \mathrm{H}$, the time constant is still $\tau_{s} \approx 0.4 \mathrm{~s}$. 


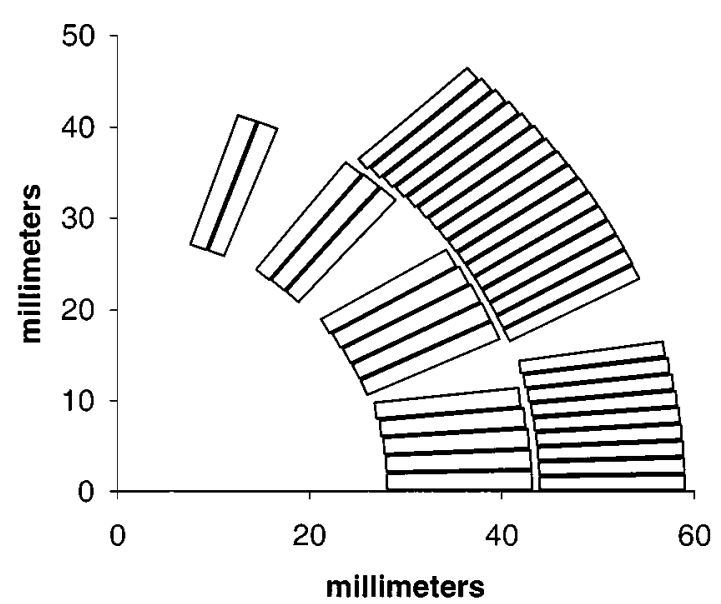

Fig. 3. The upper right quarter of the dipole winding. Together with other quarters it creates the irregular and bent stacks.

Let us now qualitatively consider a winding of the main LHC dipole shown in Fig. 3. Certainly, there is significant difference between its geometry and that of the high stack of cables. However, all blocks are stacks of cables by themselves and both inner and outer windings create a kind of high but irregular and bent stack. This means that (27) should provide a rough estimate of the time constant for the inner and outer windings. In this connection, it can be worthwhile to estimate another limit for the strand inductance. One can assume that the total dipole inductance $0.003 \mathrm{H}$ per meter per aperture can be in equal parts ascribed to 5280 strands composing one aperture. Since the length of the strand between two neighboring contacts for the inner cable is $2.1 \mathrm{~mm}$ it results in $L \approx 1.2 \cdot 10^{-9} \mathrm{H}$. As it was shown in the previous paragraph, this value is insufficient to change $\tau_{s}$ substantially.

\section{CONCLUSIONS}

An equation describing coupling currents in a flat superconducting cable subjected to the time-dependent background mag- netic field has been developed. In the uniform case, solutions of this equation allow us to find the magnetic moment of the cable subjected to harmonic magnetic field variations, the a.c. loss and the main characteristic time constant of the high stack of cables. A related estimation of the time constant for the inner winding of the main LHC dipole provides a value approximately equal to $0.3 \mathrm{~s}$ for typical contact resistances around $50 \mu \Omega$.

\section{REFERENCES}

[1] V. E. Sytnikov, G. G. Svalov, S. G. Akopov, and I. B. Peshkov, "Coupling losses in superconducting transposed conductors located in changing magnetic fields," Cryogenics, vol. 29, pp. 926-930, 1989.

[2] A. P. Verweij and H. H. J. ten Kate, "Coupling currents in Rutherford cables under time varying conditions," IEEE Trans. Appl. Supercond., vol. 3, pp. 146-149, 1993.

[3] A. A. Akhmetov, K. Kuroda, K. Ono, and M. Takeo, "Eddy currents in flat two-layer superconducting cables," Cryogenics, vol. 35, pp. 495-504, 1995.

[4] A. Devred and T. Ogitsu, "Ramp-rate sensitivity of SSC dipole magnet prototypes," in Frontiers of Accelerator Technology, S. I. Kurokawa, M. Month, and S. Turner, Eds. Singapore: World Scientific, 1996, pp. 184-308.

[5] K. H. Mess, P. Schmuser, and S. Wolff, Superconducting Accelerator Magnets. Singapore: World Scientific, 1996, pp. 102-106.

[6] A. P. Verweij, "Review on boundary-induced coupling currents," in $A d v$. in Cryog. Eng., U. B. Balachandran, Ed. New York: Plenum, 1997, vol. 44 B, pp. 1059-1068.

[7] L. Bottura, M. Breschi, F. Negrini, and P. L. Ribani, "Electromagnetic analysis of current distribution in multistrand superconducting cables," Inst. Phys. Conf. Ser., vol. 167, pp. 1191-1194, 2000.

[8] A. Akhmetov, L. Bottura, M. Breschi, and P. L. Ribani, "A theoretical investigation on current imbalance in flat two-layer cables," Cryogenics, vol. 40, pp. 627-635, 2000.

[9] A. A. Akhmetov, "Compatibility of two basic models describing a.c. loss and eddy currents in flat superconducting cables," Cryogenics, vol. 40, pp. $445-457,2000$.

[10] A. M. Campbell, "A general treatment of losses in multifilamentary superconductors," Cryogenics, vol. 22, pp. 3-16, 1982.

[11] A. A. Akhmetov, A. Devred, R. G. Mints, and R. I. Schermer, "Current loop decay in Rutherford-type cables," in Supercollider, P. Hale, Ed. New York: Plenum, 1995, vol. 5, pp. 443-446.

[12] R. Wolf, private communication, 2001.

[13] A. A. Akhmetov and S. Amet et al., "Measurement of the contact electrical resistance in the cable of MBB-A001 pre-series dipole,", internal note LHC-MTA-IN-2001-168, July 2001. 